\title{
ANHELANDO JUSTICIA EN LA ERA DEL CAMBIO CLIMÁTICO: DE LA TEORÍA A LA PRÁCTICA*
}

\section{LONGING FOR JUSTICE IN A CLIMATE CHANGED AGE: FROM THEORY TO PRACTICE}

\author{
DANIEL IGLESIAS MÁRQUEZ Y BEATRIZ FELIPE PÉREZ \\ Investigadores Asociados al Centro de Estudios de Derecho Ambiental de \\ Tarragona (CEDAT) \\ Universitat Rovira i Virgili \\ daniel.iglesias@urv.cat / beatrizirene.felipe@urv.cat
}

A partir de la Revolución Industrial, caracterizada por el incremento en el uso de combustibles fósiles, las emisiones de gases de efecto invernadero, como el dióxido de carbono y el metano, comenzaron a aumentar exponencialmente. Una de las consecuencias del aumento de estos compuestos en la atmósfera es el calentamiento global. Como explican desde el Grupo Intergubernamental de Expertos en Cambio Climático (IPCC):

"[e]l calentamiento en el sistema climático es inequívoco y, desde la década de 1950, muchos de los cambios observados no han tenido precedentes en los últimos decenios a milenios. La atmósfera y el océano se han calentado, los volúmenes de nieve y hielo han disminuido, el nivel del mar se ha elevado y las concentraciones de gases de efecto invernadero han aumentado". ${ }^{1}$

\footnotetext{
* El presente trabajo se enmarca en el proyecto de investigación DER2016-80011-P "Constitución climática global: Gobernanza y derecho en un contexto complejo", financiado por el Ministerio de Economía y Competitividad.

1 GRUPO INTERGUBERNAMENTAL DE EXPERTOS SOBRE EL CAMBIO CLIMÁTICO (2016). Cambio climático 2013. Bases físicas. Resumen para responsables de políticas. Ginebra: IPCC.
} 
Este calentamiento ha conllevado la alteración de los sistemas que conforman el planeta Tierra y de las interacciones entre los mismos, dando lugar al cambio climático, que constituye una de las mayores amenazas globales a las que se enfrenta la humanidad. Así, la variabilidad climática, junto con la exposición a fenómenos extremos (como tormentas, inundaciones, olas de frío y de calor) más complejos, intensos y frecuentes, están directamente relacionados con el aumento del hambre y de la malnutrición. ${ }^{2}$ El cambio climático también tiene repercusiones para la biodiversidad, la salud humana, la economía y el acceso al agua, entre otros.

Una de las consecuencias más alarmantes de estos impactos es el fenómeno conocido como migraciones climáticas, es decir, la situación de muchas personas que, especialmente en contextos vulnerables en el Sur global, han de abandonar sus hogares ante las amenazas que supone el cambio climático. Estas migraciones ya están teniendo lugar en varias regiones del mundo y se espera que vayan en aumento conforme se agudizan los impactos del cambio climático. ${ }^{3}$

Los riesgos y las consecuencias de estos efectos adversos afectan de manera diferenciada a los países y a las comunidades. Los países empobrecidos del Sur global, en especial la población con menos recursos económicos de estos países, son los que sufren las consecuencias del cambio climático en mayor medida, a pesar de que los países del Norte global son los responsables de la mayor parte de las emisiones históricas, tratándose, de esta manera, de un problema de justicia global. ${ }^{4}$ El agotamiento de la capacidad de la atmósfera para absorber el carbono genera así una deuda social, ambiental y económica por parte de los países del Norte global con el resto del mundo, así como con las generaciones presentes y futuras.

\footnotetext{
2 FAO; IFAD; UNICEF; OMS (2018). The State of Food Security and Nutrition in the World 2018. Building climate resilience for food security and nutrition. Roma: FAO.

${ }^{3}$ WARNER, Koko; EHRHART, Charles; SHERBININ, Alex; CHAI-ONN, Tricia. (2009). In search of shelter. Mapping the Effects of Climate Change on Human Migration and Displacement, Bonn: CARE-CIESIN-ACNUR.

4 JAMIESON, Dale (2018). "The Nature of the Problem". En: DRYZEK, John; NORGAARD, Richard; SCHLOSBERG, David (eds.). The Oxford Handbook on Climate Change and Society, Oxford: Oxford University Press.
} 
El cambio climático es una cuestión compleja en términos de justicia, tanto por los diversos impactos que genera como por los agentes de variada naturaleza que contribuyen a este problema. En este sentido, se trata de una cuestión social y ambiental que requiere de acciones - políticas y legislativas - globales para paliar sus impactos negativos y prevenirlos de manera efectiva. Como comenta Larry Lohmann al respecto "[...] es importante ver el cambio climático como la continuación y la manifestación de algunos de los mismos problemas y fuerzas sociales con los que llevamos lidiando desde hace siglos. Se trata de una cuestión de poder político, una cuestión de quién gana y quién pierde en lo que se refiere a acceso y derechos." ${ }^{5}$

La búsqueda de soluciones para prevenir y mitigar las cargas desproporcionadas que sufren los grupos de población más afectados en el Sur global, de las que son menos responsables, ha servido para acuñar el término de justicia climática como concepto que promueve la adopción de medidas más justas y equitativas (tanto en el ámbito del derecho como en el de la política), ya sea para reducir las emisiones o para impulsar la adaptación a las nuevas condiciones que genera el cambio climático. ${ }^{6} \mathrm{El}$ intento por alcanzar la justicia climática ha conformado un movimiento crítico al modelo de desarrollo económico insostenible imperante, que es altamente dependiente de los combustibles fósiles.

Entre los intentos por regular la cuestión del cambio climático destaca la Convención Marco de Naciones Unidas para el Cambio Climático (CMNUCC), ${ }^{7}$ suscrita en la Cumbre de Río de Janeiro de 1992. Este instrumento configuró el régimen global para impulsar acciones concretas por parte de la comunidad internacional. Uno de los principios más importantes en esta Convención en relación con la justicia climática es el de las responsabilidades comunes, pero diferenciadas (Principio 1, artículo 3), que se sostiene en lo explicado anteriormente: a las diferentes emisiones históricas de los países les corresponde diferente grado de responsabilidad. Este principio ya había sido

\footnotetext{
${ }^{5}$ TNI (2008). "La política del cambio climático - Entrevista con Larry Lohmann". Disponible en: $<w w w$. carbontradewatch.org/index.php?option $=$ com_content\&task=view\&id=240\&ltemid=36>.

6 BASKIN, Jeremy (2009). "The Impossible Necessity of Climate Justice?" Melbourne Journal of International Law, vol. 10, núm. 2, pp. 424-39.

7 ONU (1992). Convención Marco de las Naciones Unidas sobre el Cambio Climático. Serie de Tratados de las Naciones Unidas, vol. 1771, p. 107. Nueva York.
} 
incluido en la Declaración de Río sobre el Medio Ambiente y Desarrollo8 (Principio 7) y constituye "(...) una innovación mayor en el ámbito del Derecho internacional contemporáneo". 9

Más adelante, basándose en este Principio, la Conferencia de las Partes (COP) de la CMNUCC adoptó en 1997 el Protocolo de Kyoto, ${ }^{10}$ que estableció objetivos cuantitativos diferenciados para limitar y reducir las emisiones de gases de efecto invernadero en relación a las responsabilidades históricas de cada región. Este instrumento internacional hizo un llamado a los Estados industrializados y los Estados con economías en transición para que redujeran sus emisiones de gases de efecto invernadero al menos un $5 \%$ por debajo de los niveles de 1990 en el primer período de compromiso (2008-2012).

Mientras se esperaba a que el Protocolo de Kyoto entrara en vigor, en el año 2000 tuvo lugar una cumbre paralela a la COP 6, celebrada en La Haya. Más de 500 líderes y lideresas mundiales organizaron la Cumbre sobre Justicia Climática, que sentó las bases para el nacimiento de un movimiento popular global que buscaba soluciones para el cambio climático que, a la vez, promovieran el respeto a los derechos humanos, la equidad, los derechos laborales y la justicia ambiental, local y globalmente.

En 2002, se adoptaron los Principios de Justicia Climática de Bali, que incluyen un total de 27 principios. ${ }^{11}$ Dos años después, en 2004, se conformó el Grupo de Durban por la Justicia Climática en el que participaron organizaciones y movimientos populares de todo el mundo. Tras el encuentro, se hicieron públicos dos textos: el "Llamado a los pueblos para actuar contra el cambio climático"12 y la "Declaración de Durban sobre el Comercio de Carbono"13 en

\footnotetext{
8 Declaración de Río sobre el Medio Ambiente y el Desarrollo. Conferencia de las Naciones Unidas sobre el Medio Ambiente y el Desarrollo (CNUMAD). U.N. Doc. A/CONF.151/5/Rev.1. Rio de Janeiro, Brasil.

9 JUSTE-RUIZ, J. (2012). "El régimen internacional, normativo e institucional, en materia de cambio climático". En: Giles Carnero, Rosa (ed.). Cambio Climático, Energía y Derecho Internacional: Perspectivas de Futuro. España: Aranzadi.

10 ONU. (1997). Protocolo de Kyoto a la Convención Marco de las Naciones Unidas sobre Cambio Climático. Serie de Tratados de las Naciones Unidas vol. 2303, p. 162.

${ }^{11}$ Bali Principles of Climate Justice. Disponible en: <www.ejnet.org/ej/bali.pdf>.

12 Llamado a los Pueblos para actuar contra el Cambio Climático. Disponible en: $<w w w . c a r b o n t r a d e w a t c h . o r g / d u r b a n / c a l l c a s t . p d f>$.
} 
los que se advertía, entre otras cosas, de los peligros que suponía basar las soluciones climáticas en las leyes del mercado.

En Bali, en 2007, tuvo lugar otra conferencia en la que se fundó la asociación "Climate Justice Now" durante la COP 13. Se trata de una red de organizaciones y movimientos de todo el planeta que luchan por la justicia social, ambiental y de género. ${ }^{14}$ Asimismo, el objetivo de esta misma COP 13 fue comenzar a establecer las bases para negociar el periodo posterior a 2012 del Protocolo de Kyoto, ya que el primer periodo solo cubría hasta el año $2012 .{ }^{15}$

Un año después, en 2008, la reunión inaugural del Foro Humanitario Global se enfocó en la justicia climática ${ }^{16} \mathrm{y}$, un año más tarde, en 2009 , se organizó la "IV Cumbre de Pueblos y Nacionalidades Indígenas del Abya Yala" en la que las comunidades indígenas de toda América Latina acordaron

"construir un Tribunal de Justicia Climática que juzgue éticamente a las empresas transnacionales y los gobiernos cómplices que depredan la Madre Naturaleza, saquean nuestros bienes naturales y vulneran nuestros derechos, como el primer paso hacia una Corte Internacional sobre Derechos Ambientales". ${ }^{17}$

Este mismo año acontecieron otros hitos importantes en relación a la justicia climática: se conformó la red "Climate Justice Action" justo antes de la COP 15, en Copenhague; se celebró la conferencia "Hacia la Construcción de Alternativas en la Justicia Climática”, en San Salvador; y en Cochabamba

13 Declaración de Durban sobre el Comercio de Carbono. Disponible en: www.otrosmundoschiapas.org/index.php/temas-analisis/28-47-capitalismo/28-cambioclimatico/726-declaracion-de-durban-sobre-el-comercio-de-carbono

${ }^{14}$ Climate Justice Now! Disponible en: <https://climatejusticenow.org/>.

${ }^{15}$ En la COP 18 se logró finalmente ratificar su segundo periodo de vigencia hasta diciembre de 2020. No obstante, no todos los Estados estuvieron de acuerdo con los compromisos establecidos. Así, países como los Estados Unidos, uno de los mayores emisores, nunca ratificó el Protocolo y Canadá se retiró una vez que entró en vigor en 2005. En este sentido, el Protocolo de Kyoto no ha cumplido sus objetivos y ha sido calificado como un fracaso para alcanzar avances significativos en la lucha contra el cambio climático y las injusticias que se derivan de este fenómeno. Estenssoro, Fernando (2010). "Crisis ambiental y cambio climático en la política global: un tema crecientemente complejo para América Latina". Universum, vol. 25, núm. 2, pp. 57-77.

${ }^{16}$ Global Humanitarian Forum. 2009 Forum. Human Impact of Climate Change. Disponible en: $<$ www.gci.org.uk/Documents/GHF_2009_.pdf>.

17 CAOI. "Construyamos el Tribunal de Justicia Climática". Disponible en: $<$ www.alainet.org/es/active/30687>. 
(Bolivia) se celebró la Primera Audiencia del Tribunal Internacional de Justicia Climática, en la que se presentaron siete casos de injusticias climáticas en Latinoamérica.

En 2015, tras el fracaso en 2009 de la adopción de un nuevo acuerdo universal y jurídicamente vinculante que debía haber sido alcanzado en la COP 15 y de un largo proceso de negociaciones, ${ }^{18}$ los esfuerzos para prevenir y mitigar los efectos adversos del cambio climático adoptaron un nuevo enfoque con la adopción del Acuerdo de París, aprobado durante la COP 21. ${ }^{19}$ Este instrumento establece el objetivo de mantener el aumento de la temperatura media mundial muy por debajo de $2^{\circ} \mathrm{C}$ con respecto a los niveles preindustriales, y proseguir los esfuerzos para limitar ese aumento de la temperatura a $1,5^{\circ} \mathrm{C}$ con respecto a los niveles preindustriales (artículo 2).

Además de este objetivo al que se someten las partes, el Acuerdo de París presenta algunas novedades para el régimen internacional del cambio climático en relación a la justicia climática. ${ }^{20}$ En este sentido, el Preámbulo del Acuerdo hace referencia explícita por primera vez al concepto de justicia climática.

Aunque esta referencia es meramente declaratoria, este nuevo enfoque de justicia climática plantea un nuevo escenario para lucha contra el cambio climático y para dar respuestas justas y equitativas a las cuestiones asociadas a este fenómeno que, hasta el momento, ni el derecho ni la política internacional han podido solucionar de manera integral.

Asimismo, el Acuerdo de París también consiguió que los países del Norte global confirmaran su compromiso de ayudar a financiar los planes de adaptación. El Acuerdo establece en su artículo 4.4. una diferenciación de responsabilidades y compromisos entre los países industrializados y en vías de desarrollo, lo que conlleva que aunque todos los países deben establecer planes de control, los países desarrollados deberán liderar los esfuerzos y

\footnotetext{
18 ANTENTAS, Josep Maria; VIVAS, Esther (2010). "Justicia climática y justicia social: un mismo combate contra el capitalismo global”. Ecología política, núm. 39, pp. 103-106.

19 Informe de la Conferencia de las Partes 21 período de sesiones. París, 30 de noviembre a 11 de diciembre de 2015. Doc. FCCC/CP/2015/L.9.

20 LUCAS GARÍN, Andrea (2017). "Novedades del Sistema de Protección Internacional de Cambio Climático: el Acuerdo de París". Revista del Instituto de Estudios Internacionales de la Universidad de Chile, vol. 49, núm. 186, pp. 137-167.
} 
apoyar la lucha contra el cambio climático de los países en vías de desarrollo a través de ayuda financiera, establecer objetivos más ambiciosos o aumentar la capacidad de adaptación y mitigación del cambio climático de estos países mediante de la transferencia de tecnología (artículo 10).

Desde la doctrina legal y política basada en la justicia climática se estima que el enriquecimiento injusto de los países industriales debe reflejarse en la distribución tanto de obligaciones como de responsabilidades -legales y morales- para responder al cambio climático, así como para compensar a las generaciones presentes y futuras por las actividades generadoras de las emisiones en el pasado y para ayudar a las comunidades más vulnerables a adaptarse. No obstante, el régimen internacional del cambio climático se ha mantenido hasta la fecha carente de mecanismos que permitan responsabilizar a quienes contribuyen de manera significativa al cambio climático.

En relación con lo anterior, el movimiento por la justicia climática viene impulsando la búsqueda de mecanismos efectivos de reparación y compensación basados en los actuales sistemas de responsabilidad por daños ambientales, con el fin de que los países del Sur global puedan hacer efectiva la responsabilidad climática del Norte global. ${ }^{21}$

Las víctimas del cambio climático han recurrido a diversas instancias judiciales en búsqueda de justicia climática. En los últimos años se ha registrado un incremento en las demandas y reclamaciones interpuestas por las víctimas y las organizaciones de la sociedad civil contra los Estados, exigiendo que cumplan con sus compromisos adquiridos en materia de cambio climático. En dichos litigios se alegan violaciones a los derechos fundamentales y la equidad intergeneracional, entre otras.

A pesar de que el éxito de los litigios climáticos ha sido limitado, se han establecido algunos precedentes importantes que ejercen presión sobre los Estados para llevar acciones efectivas en la lucha contra el cambio climático. Por ejemplo, en los Países Bajos, en 2013, la ONG Urgenda, en representación de 886 particulares, interpuso una acción contra del Ministerio de

${ }^{21}$ FARBES, Daniel A. (2017) "How Legal Systems Deal with Issues of Responsibility for Past Harmful Behavior". En: MEYER, Lukas H.; SANKLECHA, Pranay (eds.). Climate Justice and Historical Emissions. Cambridge: CUP, pp. 80-106. 
Infraestructuras y Medio Ambiente (Ministerie van Infrastructuur en Milieu), exigiendo al Estado que mejorara su meta de reducción de gases de efecto invernadero con el fin de que en 2020 se redujera la cantidad de emisiones de $\mathrm{CO}_{2}$ respecto a 1990 en un $40 \%$ o, al menos, en un $25 \%$. En 2015 , en una decisión pionera, el Tribunal del Distrito de La Haya determinó que el Gobierno de los Países Bajos es responsable de asegurar que la reducción de las emisiones de $\mathrm{CO}_{2}$, ordenando así reducir las emisiones $\mathrm{CO}_{2}$ en al menos $25 \%$ para 2020 , en lugar del $16 \%$ previsto en su política ambiental..$^{22}$

La decisión fue apelada por el Gobierno de los Países Bajos, alegando la injerencia del tribunal en el contenido de las políticas gubernamentales que deberían discutirse en el Parlamento y no en sede judicial, de conformidad con el principio de separación de poderes. No obstante, en 2018, el Tribunal de Apelación rechazó las alegaciones del Gobierno y confirmó la decisión, señalando que la evaluación de las políticas que afectan el disfrute de los derechos humanos es parte de las obligaciones de los tribunales, sin afectar la separación de poderes. El Tribunal reafirmó el objetivo de reducir las emisiones $\mathrm{CO}_{2}$ en al menos $25 \%$ para 2020, no obstante, el Gabinete y el Parlamento se encargarán de establecer las medidas políticas para alcanzar dicho objetivo ${ }^{23}$ aunque, recientemente (noviembre de 2018), el Gobierno de los Países Bajos anunció que volverá a apelar la decisión y pedirá a la Corte Suprema que revise su última decisión ${ }^{24}$. Aún así, este precedente dio (y seguirá dando) lugar a casos similares en otras jurisdicciones.

Si bien la mayor parte de los litigios climáticos se han desarrollado en los países del Norte global, es muy probable que empiecen a surgir este tipo de acciones con mayor frecuencia en los Estados del Sur global. Así, ya se han emprendido en países como India, Sudáfrica, Filipinas y Pakistán. ${ }^{25}$ En Colombia, por ejemplo, recientemente se interpuso por parte de 25 niños y

\footnotetext{
22 RODRÍGUEZ GARCÍA, Noel (2016). "Responsabilidad del Estado y cambio climático: el caso Urgenda contra Países Bajos". Revista Catalana de Dret Ambiental, vol. 7, núm. 2, pp. 1-38.

${ }^{23}$ VERSCHUUREN, Jonathan (2018). "Urgenda Climate Change Judgment Survives Appeal in the Netherlands". Disponible en: <https://blog.uvt.nl/environmentallaw/?p=354>.

24 WANG, Ucilia (2018). "Dutch Government Will Again Appeal Historic Urgenda Climate Verdict". Disponible en www.climateliabilitynews.org/2018/11/19/urgenda-climate-ruling-appealnetherlands/.

25 PNUMA (2017). El estado del litigio en materia de cambio climático. Una revisión global. Nairobi: PNUMA.
} 
jóvenes una acción de tutela histórica contra la deforestación en la Amazonía en la que la Corte Suprema de Justicia ordenó al Gobierno expedir en cuatro meses un plan eficaz para detener la deforestación en la Amazonia, así como construir un Pacto Intergeneracional por la Vida del Amazonas Colombiano, todo en conjunto con las y los jóvenes peticionarios y las entidades y organizaciones relevantes. Asimismo, la sentencia también reconoce a la Amazonía como sujeto de derechos y ordena a los alcaldes y corporaciones autónomas de la región a cumplir sus deberes contra la deforestación. ${ }^{26}$

En relación a lo anterior, desde la perspectiva de la justicia climática el cambio climático se trata de una cuestión de derechos humanos. En esta línea de ideas, en la sentencia del caso Ashgar Leghari and the Federation of Pakistan et al. se señaló que "[c]limate Justice links human rights and development to achieve a human-centered approach, safeguarding the rights of the most vulnerable people and sharing the burdens and benefits of climate change and its impacts equitably and fairly". ${ }^{27}$

Así, está ampliamente reconocido que el cambio climático tiene consecuencias claras e inmediatas para el pleno disfrute de los derechos humanos, en especial el derecho a la vida, a un nivel de vida adecuado y al disfrute del más alto nivel posible de salud. En el marco del trabajo del Relator Especial de las Naciones Unidas sobre los derechos humanos y el medio ambiente, John Knox, se determinó que los efectos negativos previsibles del cambio climático en el disfrute de los derechos humanos entrañan para los Estados la obligación de adoptar medidas destinadas a protegerlos. ${ }^{28}$

El Derecho internacional de los derechos humanos viene a reforzar el actual movimiento de justicia climática. Así, el Preámbulo del Acuerdo de París señala expresamente que el cambio climático es un problema de toda la humanidad y que, al adoptar medidas para hacerle frente, las Partes deben respetar, promover y tener en cuenta sus respectivas obligaciones relativas a los

${ }^{26}$ Corte Suprema de Justicia de Colombia. STC4360-2018. Radicación no. 11001-22-03-0002018-000319-01.

27 Case no. W.P. No. 25501/2015. Disponible en: $<$ www.pja.gov.pk/system/files/2018LHC106.pdf>.

${ }^{28} \mathrm{CDH}$ (2016). Informe del Relator Especial sobre la cuestión de las obligaciones de derechos humanos relacionadas con el disfrute de un medio ambiente sin riesgos, limpio, saludable y sostenible. Doc. A/HRC/31/52. 1 de febrero de 2016. 
derechos humanos. ${ }^{29}$ Por tanto, las obligaciones de los Estados en materia de derechos humanos se extienden a las decisiones relativas a la protección del clima, incluyendo aquellas relacionadas con las medidas de mitigación y adaptación. Esto implica que los Estados deben adoptar un marco jurídico e institucional que preste asistencia a quienes estén bajo su jurisdicción para adaptarse a los efectos inevitables del cambio climático. ${ }^{30}$

El incumplimiento de la obligación de los Estados de proteger los derechos humanos para que el cambio climático no los vulnere ha motivado acciones ante los tribunales internacionales de derechos humanos por la responsabilidad de un Estado por violaciones de derechos humanos asociados al cambio climático.

El primer caso en el que se alegó la responsabilidad de un Estado por las violaciones de derechos humanos derivadas de los impactos del cambio climático es la petición que lideró Sheila Watt-Cloutier, que en 2004, con apoyo de la Conferencia Circumpolar Inuit, el Center for International Environmental Law (CIEL) y EarthJustice en nombre de todas las regiones árticas de Estados Unidos y Canadá ante la Comisión Interamericana de Derechos Humanos (CIDH). En este caso se alegaba que Estados Unidos es uno de los mayores responsables del cambio climático debido a la acumulación de emisiones históricas y actuales de gases de efecto invernadero, argumentándose que se estaban violando tanto los derechos de carácter material como inmaterial de los pueblos indígenas. ${ }^{31} \mathrm{La} \mathrm{CIDH}$ no admitió el caso, argumentando que la información que se proveía era insuficiente para demostrar un vínculo de causalidad, sin embargo, accedió a celebrar una audiencia pública en 2007.

En 2013, la organización Earthjustice, en nombre del pueblo de Athabaskan que habita en el Ártico, interpuso una petición ante la CIDH. En ella se alegó que Canadá ha fallado en su obligación de regular y limitar las emisiones de

\footnotetext{
29 Informe de la Conferencia de las Partes 21 período de sesiones. París, 30 de noviembre a 11 de diciembre de 2015. Doc. FCCC/CP/2015/L.9.

30 Informe del Relator Especial sobre la cuestión de las obligaciones de derechos humanos relacionadas con el disfrute de un medio ambiente sin riesgos, limpio, saludable y sostenible, de 1 de febrero de 2016, A/HRC/31/52.

${ }^{31}$ Veáse, NIEHUSS, Juliette (2005). "Inuit Circumpolar Conference v. Bush Administration: Why the Arctic Peoples Claim the United States' Role in Climate Change has violated their Fundamental Human Rights and Threatens their Very Existence". Sustainable Development Law \& Policy, vol. 5, núm. 2, pp. 66-67.
} 
carbono negro, consideradas como una de las causas del cambio climático. Por ende, se generan impactos directos en el Ártico que afectaban la salud, cultura, propiedad y medios de subsistencia del pueblo de Athabaskan, violando así derechos contemplados en la Declaración Americana de los Derechos y Deberes del Hombre. ${ }^{32}$ Esta petición está aún pendiente de resolverse en la $\mathrm{CIDH}$. Si bien puede llegar a ser rechazada por la $\mathrm{CIDH}$ como casos en anteriores, existe un importante avance claridad el nexo causal entre la acción del Estado y las violaciones de derechos humanos a causa del derretimiento del Ártico por las emisiones de carbono negro. ${ }^{33}$

La oleada del litigio climático no solo arremete contra los Estados, sino también, aunque en menor medida, contra las empresas, haciendo hincapié en que el objetivo de mantener el aumento de la temperatura media mundial muy por debajo de $2^{\circ} \mathrm{C}$ es responsabilidad de los diversos agentes públicos y privados que contribuyen al cambio climático. ${ }^{34}$ Los avances en la ciencia del cambio climático han podido determinar y cuantificar de manera cada vez más exacta en qué medida las actividades de las empresas contribuyen al cambio climático. El estudio de Heede, por ejemplo, mapea las emisiones acumuladas de las 90 mayores empresas productoras de carbono (Carbon Majors), concluyendo que su contribución al cambio climático es de casi dos tercios del global de las emisiones de gases de efecto invernadero entre 1854 y 2010.35

Las víctimas de los efectos adversos del cambio climático han aprovechado la coyuntura internacional y los avances científicos para interponer acciones contra los Carbon Majors con el fin de exigirles que prevengan sus contribuciones al cambio climático o compensen los daños resultantes del cambio climático. Por ejemplo en 2015, Saul Luciano Lliuya, un agricultor de la región andina de Perú, interpuso una demanda en contra de uno de los Carbon

32 EARTHJUSTICE (2013). "Petition to the Inter-American Commission on Human Rights Seeking Relief from Violations of the Rights of Arctic Athabaskan Peoples Resulting from Rapid Arctic Warming and Melting Caused by Emissions of Black Carbon by Canada". Disponible en: https://earthjustice.org/sites/default/files/AAC_PETITION_13-04-23a.pdf.

33 MCCRIMMON, Don (2016). "The Athabaskan Petition to the Inter-American Human Rights Commission: using human rights to respond to climate change". The Polar Journal, vol. 6, núm. 2, pp. 398-416.

34 En este sentido, el Acuerdo de París fomenta la participación de actores no estatales, atribuyendo a las empresas un rol más activo para la reducción de emisiones de GEI.

35 HEEDE, Richard (2013). "Tracing Anthropogenic Carbon Dioxide and Methane Emissions to Fossil Fuel and Cement Producers, 1854-2010". Climatic Change, vol. 122, num. 1-2, pp. 229241. 
Majors, la empresa alemana RWE. En la demanda se alega que su hogar en Huaraz, al pie de la laguna Palcacocha está seriamente amenazado por el potencial colapso de dos glaciares dentro del lago que causarían una inundación de grandes dimensiones como consecuencia del calentamiento global al que la empresa ha contribuido sustancialmente. ${ }^{36}$

A esta lista de acciones contra los Carbon Majors se suman también los gobiernos y autoridades locales en busca de una compensación por los costos que han tenido que asumir para hacer frente a los graves episodios meteorológicos y a la elevación del nivel del mar. En 2018, la Ciudad de Nueva York demandó a cinco de los principales Carbon Majors, entre ellos, las empresas ExxonMobil, Shell, BP, Chevron and Conoco-Phillips. ${ }^{37}$ Este tipo de litigio pone mayor presión sobre las empresas, ya que a diferencia de otros casos, estos actores gubernamentales cuentan con más recursos y experiencia en el litigio, superando así la asimetría de recursos y otros obstáculos que pueden enfrentar algunas personas afectadas en búsqueda de justicia climática, especialmente aquellas del Sur global. Por tanto, al menos por el momento, tienen mayor posibilidad de influir en el comportamiento y estrategias de las empresas en relación con el cambio climático.

En este contexto de avances teóricos y prácticos en materia de justicia climática se sitúa este segundo monográfico de la Revista Catalana de Dret Ambiental (RCDA), cuyo objetivo es recopilar algunas de las comunicaciones presentadas durante el segundo Tarragona International Environmental Law Colloquium (TIEC), un evento organizado conjuntamente por el Centro de Estudios de Derecho Ambiental de Tarragona (CEDAT) de la Universidad Rovira i Virgili y la Asociación de Alumnos y Exalumnos de Derecho Ambiental de Tarragona (AAEDAT). ${ }^{38}$

Esta segunda edición del TIEC, que tuvo lugar en mayo de 2017, llevaba por título "Longing for Justice in a Climate-Changed World: From Theory to

\footnotetext{
36 KÜPER, Stefan (2017). Historic breakthrough with global impact in "climate lawsuit". Disponible en: <https://germanwatch.org/en/14795>.

${ }^{37}$ City of New York v. BP P.L.C. et al (1:18-cv-00182), New York Southern District Court.

38 Véase: II Tarragona International Environmental Law Colloquium: Longing for Justice in a Climate-Changed World: From Theory to Practice. Abstract Proceedings. Disponible en: $<$ https://tiecolloquium.files.wordpress.com/2015/11/abstract-proceedings-2017.pdf>.
} 
Practice" 39 y en ella se contó con la presencia de tres oradores principales: la Dra. Carmen G. González (Seattle University), Dr. Duncan French (University of Lincoln) y Dr. Jamie Benidickson (University of Ottawa) que enriquecieron el debate de las y los jóvenes investigadores a lo largo del Coloquio. El presente número monográfico de la RCDA recopila cinco contribuciones en las que se plantean cuestiones relacionadas con la justicia climática desde diferentes perspectivas.

La primera contribución ("Multi-level climate governance: polycentricity and local innovation"), elaborada por Ernani Contipelli, cuestiona cómo el enfoque policéntrico puede ser aplicado a la gobernanza climática multinivel en la que se tienen en cuenta los diferentes niveles de gobierno, desatancando especialmente la importancia de la innovación local. En este estudio se señala, además, la relevancia de la innovación local como método de control de la crisis climática global a través de actividades colectivas que aseguren y mantengan los niveles de integridad y cooperación entre los diferentes actores y las instituciones.

En el segundo trabajo ("Law of the sea and environmental law acting together: experience of laying submarine cable in the Arctic"), Daria Shvets se centra en los impactos ambientales que supone colocar cables en el fondo marino. Concretamente, se centra en el proyecto que prevé colocar el primer cable submarino internacional, cuya mayor parte se establecerá en el Ártico, región especialmente sensible y que, como se comentó anteriormente, está viéndose seriamente afectada por el cambio climático. La autora examina el régimen jurídico internacional de los cables submarinos en el contexto de este proyecto y se plantea la aplicabilidad del principio de precaución y del Código Polar de la Organización Marítima Internacional como garantías de protección de este ecosistema.

Isabel Vilaseca Boixareu y Jordi Serra Calvó, en la tercera contribución ("Separación de poderes y cambio climático: un análisis de la cuestión a través de la jurisprudencia climática de Estados Unidos") abordan el litigio climático como vía para presionar a los Estados e incidir en sus políticas climáticas. Los

39 Véase: "Tarragona International Environmental Law Colloquium". Disponible en: <https://tiecolloquium.com/past-editions/tiec-2017/>. 
autores cuestionan la separación de poderes (especialmente entre los poderes políticos y el poder judicial) como obstáculo para el éxito de estos litigios, centrándose en la jurisprudencia climática de Estados Unidos.

Marina Rodríguez Beas, por su parte, analiza en el cuarto trabajo ("La incidencia del Acuerdo de París en las políticas públicas catalanas frente al cambio climático: la Ley 16/2017, de 1 de agosto, de Cambio Climático") cómo el Acuerdo de París y las iniciativas climáticas impulsadas por la Unión Europea afectan a la formulación y al desarrollo de las políticas públicas catalanas en materia de cambio climático. La autora se centra en la Ley 16/2017, de 1 de agosto, de cambio climático, analizando, entre otros, los objetivos de la misma en materia de mitigación y de adaptación.

Finalmente, en la última contribución ("Estudio de la Ley Catalana 16/2017, de 1 de agosto, de Cambio Climático, y comparativa con otras iniciativas legislativas estatales. Análisis de los instrumentos que propone. Un modelo innovador y ambicioso"), Aitana de la Varga Pastor, se centra también en la Ley catalana 16/2017, de 1 de agosto, de cambio climático pero analiza de manera más detallada las medidas previstas para las distintas políticas sectoriales y los instrumentos fiscales, como son los impuestos ambientales que contempla. Asimismo, aborda los artículos que han sido impugnados mediante recurso de inconstitucionalidad. La autora también compara el proceso legislativo catalán con el de otras comunidades autónomas que han seguido el laborioso camino de legislar en materia de cambio climático.

De todo lo anterior puede extraerse que nuestro objetivo es ir un paso más allá en el estudio de la justicia climática, abordando un amplio abanico de temas, internacionales y locales, desde el Ártico, a los Estados Unidos y Cataluña. Este monográfico no se limita a un estudio descriptivo o teórico del tema tratado en el segundo TIEC sino que presenta casos prácticos, actuales e innovadores. Los autores y autoras mantienen una perspectiva crítica y constructiva en todo momento que se puede apreciar en los trabajos y que va en línea con el espíritu del Coloquio. Esperamos con este monográfico y con las siguientes ediciones deI TIEC continuar aportando nuevas ideas en el marco del Derecho ambiental y contribuir a promover la justicia climática. 